\title{
Fuzzy-assisted social-based routing for urban vehicular environments
}

\author{
Rashid Hafeez Khokhar ${ }^{*}$, Rafidah Md Noor ${ }^{1}$, Kayhan Zrar Ghafoor ${ }^{2}$, Chih-Heng Ke ${ }^{3}$ and Md Asri Ngadi
}

\begin{abstract}
In the autonomous environment of Vehicular Ad hoc NETwork (VANET), vehicles randomly move with high speed and rely on each other for successful data transmission process. The routing can be difficult or impossible to predict in such intermittent vehicles connectivity and highly dynamic topology. The existing routing solutions do not consider the knowledge that behaviour patterns exist in real-time urban vehicular networks. In this article, we propose a fuzzy-assisted social-based routing (FAST) protocol that takes the advantage of social behaviour of humans on the road to make optimal and secure routing decisions. FAST uses prior global knowledge of real-time vehicular traffic for packet routing from the source to the destination. In FAST, fuzzy inference system leverages friendship mechanism to make critical decisions at intersections which is based on prior global knowledge of realtime vehicular traffic information. The simulation results in urban vehicular environment for with and without obstacles scenario show that the FAST performs best in terms of packet delivery ratio with upto $32 \%$ increase, average delay $80 \%$ decrease, and hops count $50 \%$ decrease compared to the state of the art VANET routing solutions.
\end{abstract}

\section{Introduction}

Recently, the social-based networks have been built to bring different groups of people within range for potential communication. Such social-based networks are not only used to connect the computers for global communications network but it can also be used to connect vehicles in urban environments. Social-based routing in Vehicular Ad hoc NETwork (VANET) is attracted the attention of research community where the traffic information that behaviour patterns exist allow us to make better routing decisions. VANET provides the ability for vehicles to communicate wirelessly among nearby vehicles and road-side wireless sensors to transfer information for safe driving, dynamic route planning, mobile sensing and in-car entertainment. Existing VANETs routing protocols, for example, GPSR [1], GPCR [2], LOUVRE [3], geographical greedy traffic-aware routing (GyTAR) [4], RBVT-R [5], GeoCross [6] and ReTARS [7], only work well in cooperative urban environments. Currently, the vehicles have short radio communication range from 300 to $1000 \mathrm{~m}$ based on IEEE 802.11p, and

\footnotetext{
* Correspondence: rashid@fsktm.um.edu.my

${ }^{1}$ Faculty of Computer Science and Information Technology, University of Malaya, 50603 Lembah Pantai, Kuala Lumpur, Malaysia

Full list of author information is available at the end of the article
}

VANET routing protocols need more vehicles to transfer data to make one-one communications across wider area. Consequently, it is necessary to develop efficient routing protocols for growing vehicular networks.

Geographical routing protocols $[1,2,4,8-11]$ are the well-suited protocols for VANETs environments. These protocols use Global Positioning System (GPS) to locate nodes on the map instead of establishing routes to forward data packets from source to the destination through intermediate nodes (neighbors). Figure 1a illustrates the routing strategy in these routing protocols in ideal urban scenario with moderate, low or high mobility. The source node $S$ first transmits the message to its neighbor nodes using greedy or geographical forwarding method in the street and perimeter probing at intersections. The message has been reached at intersection $I_{2}$ through route $R_{1}$ to $R_{2}$ where the decision-making node $N$ takes an important decision. The node $N$ selects route $R_{4}$ and finally reaches at destination node $D$ through $R_{5}$. However, Figure $1 \mathrm{~b}$ depicts the two problems arise when these protocols are implemented on real-world urban traffic scenario. First, it might be possible that there is no node at intersection $I_{2}$ within the period of Time-to-Live (TTL) to make an important decision. In this case, the message is forwarded to next

\section{国}




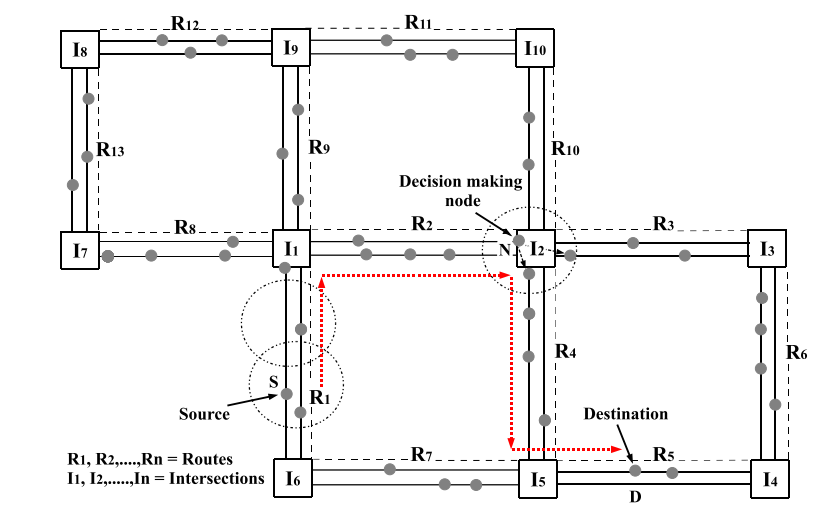

(a) Routes established in ideal city scenario

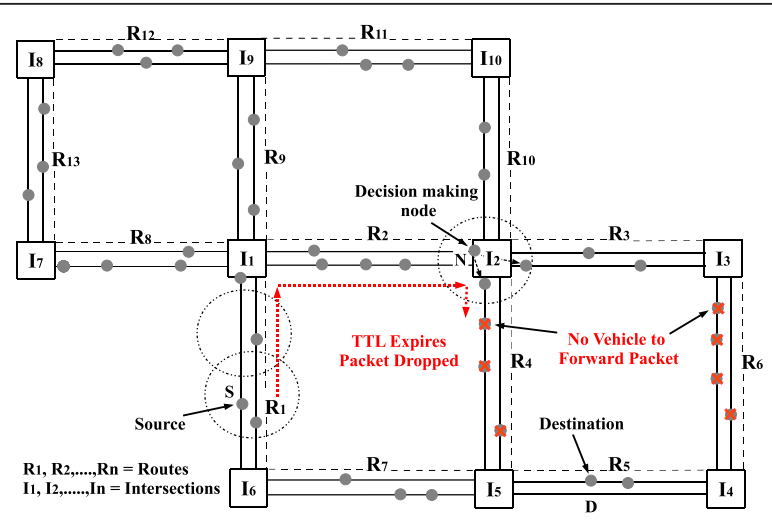

(b) Routes failure in real-world city scenario

Figure 1 Routing strategy in existing VANET routing protocols without prior global knowledge

available node away from the intersection. Second, if there is no vehicle on next routes, $R_{4}$ and $R_{6}$, it can cause unnecessary traffic overhead in the network and longer delays for packets.

Another major problem in VANET routing protocols is the dead-end roads that may cause many data packets dropped, failure notification increases significantly, low delivery ratios and fail to find shortest path. As illustrated in Figure 2, in most of the existing geographical routing protocols the message forwards to nodes $A, B$ and $C$ on a dead-end road which is the shortest path from $S$ to $D$. However, the message should follow the dotted path as depicted in Figure 2. Greedy distributed spanning tree routing (GDSTR) [12] proposed to find shorter routes and generates less maintenance traffic if greedy forwarding fails at the dead-end roads. GDSTR creates and maintains hull trees to guide packets around dead-end roads instead of using planarization algorithm.

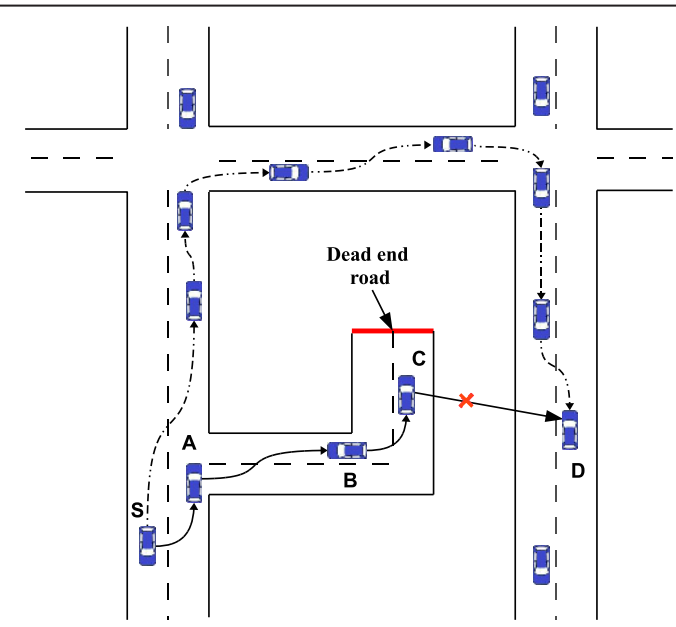

Figure 2 Dead-end roads can cause unnecessary overhead in VANET.
The simulation results have shown that GDSTR incurs significantly lower overhead than protocol proposed in [13]. A geo-proactive overlay routing called Landmark Overlays for Urban Vehicular Routing Environments (LOUVRE) [3] proposed to create an overlay links on top of an urban topology. In LOUVRE, the nodes at intersections are defined as landmark and the overlay links are only possible if there is enough traffic density between intersections. LOUVRE's guaranteed multi-hop routing is a suitable way to avoid dead-end roads. Jerbi et al. [4] also proposed an intersection-based Greedy Traffic-Aware Routing (GyTAR) protocol to find best routes in urban environments. GyTAR creates routes from source to destination based on sequence of connected intersections. Two parameters including change in vehicular traffic information and the remaining distance from the destination are used to define a best route. GyTAR also used an improved greedy forwarding mechanism to forward data packet on the road segments. However, if there is no node at intersection, then the packet cannot be forwarded and the performance of LOUVRE and GyTAR affects as data packet dropped and higher end-to-end delay. In another attempt, Nzouonta et al. [5] proposed a reactive-based VANET routing protocol called Road-Based using Vehicular Traffic information-Reactive (RBVT-R), which creates paths containing the successions of road intersections with high probability and network connectivity using real-time vehicular traffic information. RBVT-R works well in cooperative environment. However, they did not considered anonymity issues during packet routing in harsh vehicular network. In addition, static weights used in RBVT-R cannot implement on real VANET urban environment where network and traffic conditions dynamically change.

In this article, we propose a FAST protocol to make dynamic routes based on prior global knowledge using 
friendship mechanism. Instead of simply forwarding the message to next available node towards destination like in existing VANET routing protocols, we use more reliable approach with the help of social relations of vehicles for optimal routing. The route message is forwarded to next available node in streets if and only if the intersection is far away from the node. In FAST, the packet career node at intersection plays a key role to select the best next road segments and leverages fuzzy inference system to make reliable and secure routing towards destination. The rest of the article is organized as follows. Section 2 presents the proposed FAST protocol with examples from urban environment. In Section 3 , we evaluate the performance of FAST by comparing with some existing VANET routing protocols and the article concludes with some future studies in Section 4.

\section{Proposed fuzzy-assisted social-based routing (FAST) protocol}

We propose the FAST protocol that creates routes dynamically for optimal routing in urban vehicular environments. In FAST, the prior global knowledge of real-time vehicular traffic is used to create routes dynamically. The basic idea behind FAST is that first source node broadcasts a short message with secure ID to the neighbor nodes. Source node determines the types of nodes when it confirms this node in the list of friends or friends-of-friends. The nodes that are not in the friends list will automatically be discarded. The source node may have more than one friend, in that case, a node which is closer to destination forwards the message to next available node. But, if there is no next node available at intersection to forward the data packet then the current node in the street will hold the message if and only if it can reach at intersection before TTL expires, otherwise the message is forwarded to next available node in the same street. We compare TTL with the time a node takes to reach at intersection. The time a node takes to reach at intersection is determined as time $=$ distance/speed. If the node can reach at intersection before TTL expires, this node becomes a decision-making node where it uses prior global knowledge of real-time vehicular traffic to forward message to the best suitable route towards destination. The decisionmaking node uses traffic-density information based on friends, friends-of-friends, and non-friends information on each road segment and implement fuzzy inference system to determine best route towards destination. In the following sections, we explain the steps involved in the design of FAST protocol.

\subsection{Friendship mechanism}

The prior global knowledge of real-time traffic is determined by the node-density information in urban environment. As illustrated in Section 1, the importance of prior global knowledge and how the existing routing protocols are fail to find next hop if there are not enough nodes on next road segments. We use this information to propose a friendship mechanism that will speed up the route creation process of trusted route towards destination. The real-time traffic information is divided into three classes of mutual relationships such as friends, friends-of-friends and non-friends. The friendship mechanism is not proposed to design a fully operational intrusion detection system (IDS) for vehicular networks. The purpose is to show that how the social relationships between vehicles can be used for significance performance of VANET routing protocols. We have implemented only simple operational misuse and anomaly detection engines based on existing works in $[14,15]$. We have assumed that a pair of direct friends or friends-of-friends who have mutual trust with each other can communicate. The performance of friendship mechanism in highly dynamic VANET routing protocol is reduced, if each possible security relationship fully owned by any two vehicles. It requires a lot of efforts if each vehicle checks the secure relationship with other vehicles. The proposed friendship mechanism is simple yet efficient in the sense of exchange data packets with other trusted vehicles.

We have considered three types of relationships including direct friends, indirect friends (friends-offriends) and non-friends. The vehicles are used by humans and their behaviours are based on social network. In direct friendship, the vehicles may establish relations using personal judgement in daily life experiences. As illustrated in Figure 3, the nodes can start establish mutual relation in office and can be later direct friends using Facebook, Twitter, Google+, LinkedIn, etc. The nodes can also establish their relations on some other places such as residential area, playground, shopping mall, etc. On the other hand, indirect friendship is based on the good reputation of other vehicles. There are some advantages of these types of friendship in terms of security, packet delivery ratio (PDR) and average delay. Most of existing security solutions are associated with the authentication mechanisms, which usually require expensive cryptography and an assumption of a central authority. In addition, almost all of the existing works lack one important feature, which is no collaborative effort among nodes to create a trusted vehicular community. The creation of a trusted vehicular network is important to ensure an efficient Intelligent Transportation System (ITS).

Furthermore, in trusted vehicular networks, the data packets can be forwarded to friends and friends-offriends without any detailed security check for high PDR and lower average delay. However, the average delay 


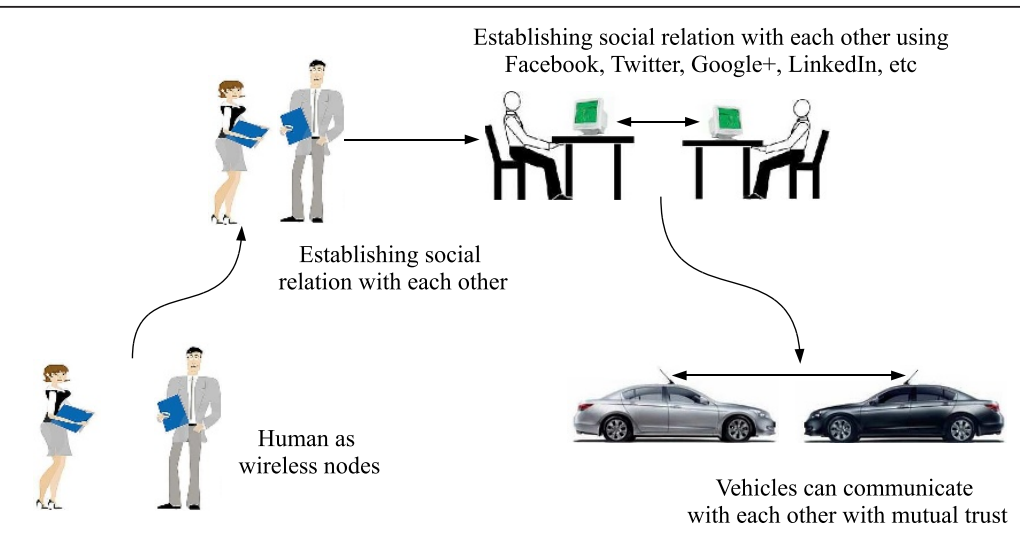

Figure 3 Social relation establishment between vehicles based on personal experiences.

may increase if there is less number of direct or indirect friends on the road. Although, the non-friends vehicles cannot directly be added in the list of friends and friends-of-friends. The new node can join the network after establishing the mutual trust with friends or friends-of-friends. There are two possible methods to create a new set of friend nodes including real-world experience and reputation of new node. Initial trust based on a real-world friendship is more relevant than that established based on nodes' experiences at the early stages of the proposed framework implementation. This is because in such situation, each node is very unlikely to have sufficient knowledge/experience about other nodes, thus will not be able to rate other nodes' reputations. Initial trust based on reputation is more suitable at the later stages when sufficient experiences have been gathered. Perhaps the combination of the two methods could result in a better performance. However, for simplicity, only initial trust based on a real-world friendship is implemented in the experiment to show how a trusted community could be created in vehicular urban environments. The direct friendships will be exchanged between trusted friends to create a new set of friend nodes, namely indirect friends (friends-of-friends). However, if a node does not want to join social network will be considered as non-friends node.

\subsection{Design of fuzzy logic decision making system}

It has discussed in Section1 that the vehicles move on the roads with high speed in VANET and node-density information frequently change from sparse to dense and vice versa. Optimal decision plays an important role for efficient data packet forwarding in highly dynamic VANET environments. Artificial intelligence techniques such as fuzzy logic perform well in classification and decision-making systems $[16,17]$. We have used the fuzzy logic system to make better decision at intersection for meaningful performance of the proposed FAST protocol. The design of fuzzy logic decision-making system consists of input membership functions and a set of fuzzy rules. The basic idea is taken from human brain, which simulates the interpretation of uncertain sensory information [18]. In this study, it is applied on number of friends, friends-of-friends, and non-friends which is based on efficient arrangement of metrics (percentages of friends, friends-of-friends and non-friends). In this case, the packet carrier node does not know which path is more efficient and secure (based on the rate of friends) for the significance routing. Thus, the fuzzy logic decision-making system offers an efficient solution for this type of uncertain situation.

Figure 4 shows the steps involved in the design of fuzzy logic decision-making system such as fuzzification of input \& output, fuzzy inference engine, and defuzzification. Firstly, the input and output variables and their membership functions are determined. Secondly, important step is to define the fuzzy rules based on input and output variables. This is followed by a group of rules used to represent inference engine (knowledge base) for articulating the control action in linguistic form. The following sections explain the input parameters used in fuzzy inference system.

\subsubsection{Fuzzification of inputs and outputs}

Three input parameters are fuzzified including friends, friends-of-friends, and non-friends as illustrated in Figure 5. The membership functions namely Sparse, Medium and Dense are used to represent the traffic density of friends, friends-of-friends, and non-friends. The selection of friends, friends-of-friends, and non-friends membership functions can be derived based on experience as well as trial-and-error of the application requirement, thus, the range should be between 0 and 1 . The actual reason to select this range is that a node might not have same list of friends 0 or all nodes have friends list 1 in the same path to the specified destination. When nodes are establishing routes, the values of friends may vary 


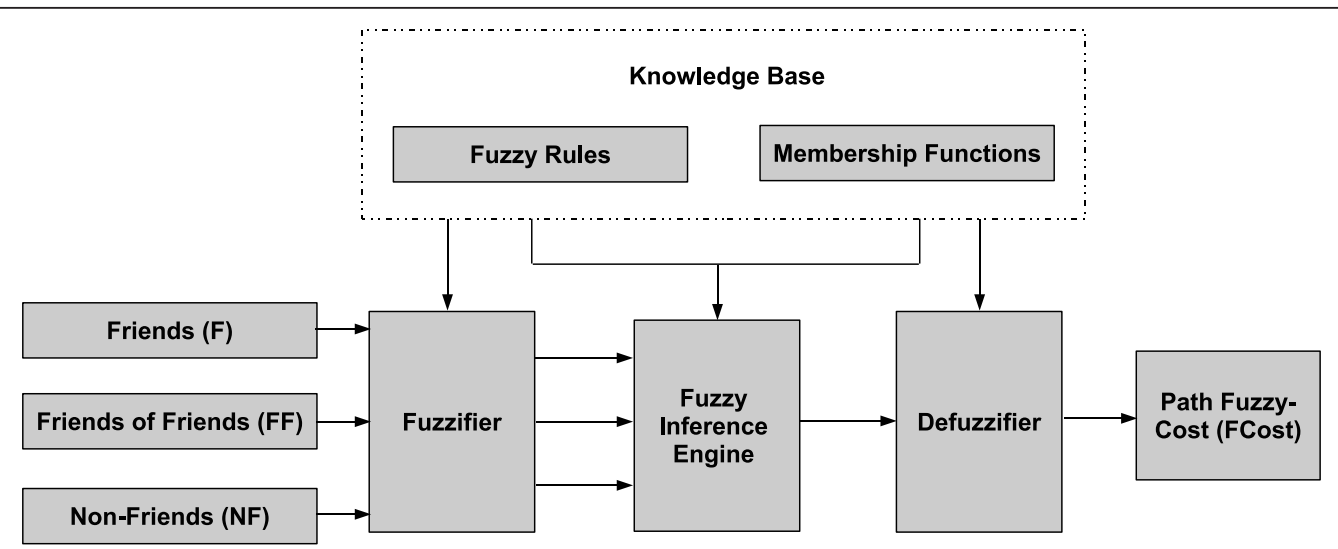

Figure 4 Fuzzy logic components (fuzzification, inference engine, and defuzzification) to rank available paths.

from minimum to maximum. So, the friendship value is selected in reply to the percentage variation intelligently integrated with the status of the nodes.

The output fuzzy cost is configured to a range between 0 and 1; the greater this value, the more efficient and optimal route will be. We have also used computationally efficient triangular functions as membership functions. The efficient design of membership function has a positive impact on the performance of fuzzy decision-making process.

\subsubsection{Fuzzy inference engine}

In this step, we develop a set of rules using expert knowledge about meaningful performance of FAST protocol. The knowledge-based fuzzy rules are designed to integrate the inputs and outputs variables which are based on careful understanding of traffic patterns of vehicular urban networks. We have defined 27 fuzzy rules to design fuzzy inference decision-making system, as shown in Table 1. Each rule consists of a IF part, a logical connection and a THEN part. The IF conditions

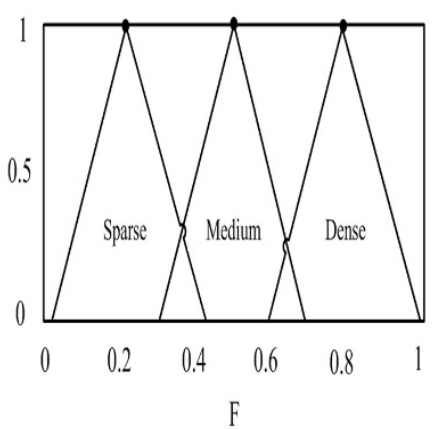

(a) Input variable friends

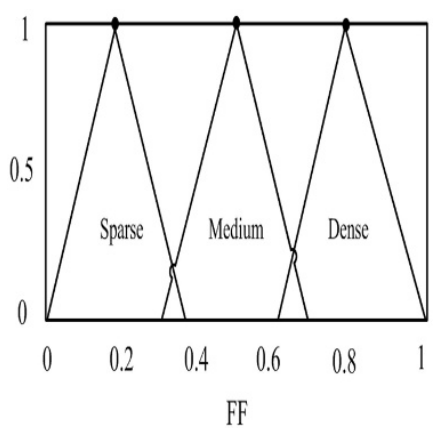

(b) Input variable friends-offriends

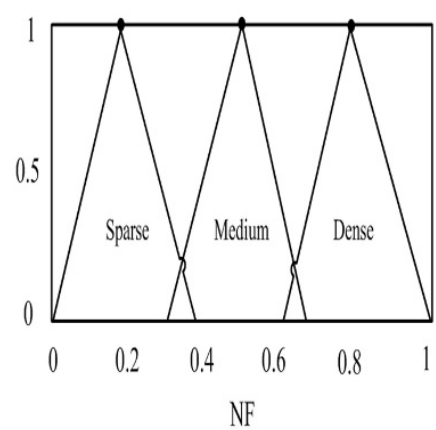

(c) Input variable non-friends

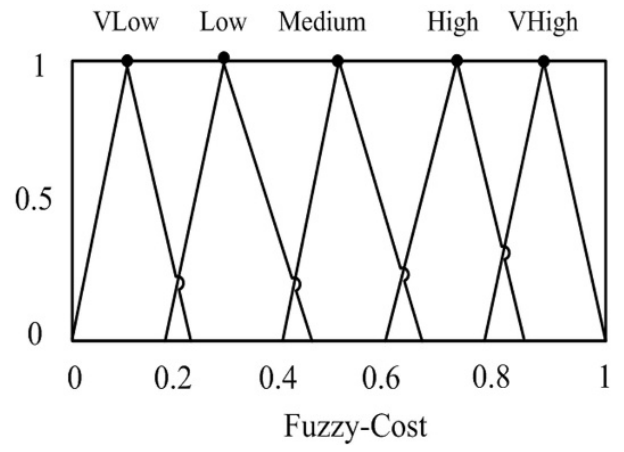

(d) Output variable fuzzy cost

Figure 5 Fuzzification of three input variables (friends, friends-of-friends, and non-friends) and output variable (fuzzy cost) 
Table 1 Knowledge structure based on fuzzy rules

\begin{tabular}{|c|c|c|c|c|c|c|c|c|c|}
\hline \multirow[b]{2}{*}{ Rule } & \multirow[b]{2}{*}{$\mathrm{F}^{*}$} & \multicolumn{2}{|l|}{ IF } & \multicolumn{3}{|c|}{ THEN } & \multicolumn{2}{|c|}{ IF } & \multirow{2}{*}{$\begin{array}{l}\text { THEN } \\
\text { FCost }\end{array}$} \\
\hline & & $\mathrm{FF}^{*}$ & $\mathrm{NF}^{*}$ & FCost* & Rule & $\mathrm{F}$ & $\mathrm{FF}$ & $\mathrm{NF}$ & \\
\hline 1 & Sparse & Sparse & Sparse & VLow & 15 & Medium & Medium & Dense & Low \\
\hline 2 & Sparse & Sparse & Medium & Low & 16 & Medium & Dense & Sparse & High \\
\hline 3 & Sparse & Sparse & Dense & VLow & 17 & Medium & Dense & Medium & High \\
\hline 4 & Sparse & Medium & Sparse & Low & 18 & Medium & Dense & Dense & Medium \\
\hline 5 & Sparse & Medium & Medium & Low & 19 & Dense & Sparse & Sparse & VHigh \\
\hline 6 & Sparse & Medium & Dense & Low & 20 & Dense & Sparse & Medium & Medium \\
\hline 7 & Sparse & Dense & Sparse & Medium & 21 & Dense & Sparse & Dense & Medium \\
\hline 8 & Sparse & Dense & Medium & Medium & 22 & Dense & Medium & Dense & High \\
\hline 9 & Sparse & Dense & Dense & Low & 23 & Dense & Medium & Medium & High \\
\hline 10 & Medium & Sparse & Sparse & Medium & 24 & Dense & Medium & Sparse & VHigh \\
\hline 11 & Medium & Sparse & Medium & Medium & 25 & Dense & Dense & Sparse & VHigh \\
\hline 12 & Medium & Sparse & Dense & Low & 26 & Dense & Dense & Medium & High \\
\hline 13 & Medium & Medium & Sparse & High & 27 & Dense & Dense & Dense & High \\
\hline 14 & Medium & Medium & Medium & High & & & & & \\
\hline
\end{tabular}

$\mathrm{F}$, friends; ${ }^{*} \mathrm{FF}$, friends-of-friends; ${ }^{*} \mathrm{NF}$, non-friends; ${ }^{*} \mathrm{FCost}$, fuzzy-cost.

are built using predicates, and a logical connection is used to connect antecedent and consequent parts, whereas the THEN statement gives a degree of membership function that befits the fuzzy variables involved. We have designed fuzzy rules to give highest rank to the route which has dense number of friends and friends-offriends. Thus, our FAST favours secure and fully connected route towards packet's destination. For instance, in the case where F is 0.842 and FF is 0.137 and NF is 0.103, then FCost is 0.893 . The path has this fuzzy cost because of its high rate of friends and the sparse distribution of non-friend vehicles. It means that our fuzzy inference system uses a trade-off decision between parameters (friends, friends-of-friends, and non-friends) to adaptively tune the cost of each path to the specified destination. In addition, Figures 6 and 7 depict the relation between input and output variables. The trend shows that the value of output fuzzy cost increases when the value of F and FF are increasing. Thus, our fuzzy inference system could increase fuzzy cost as number of friends per route increases.

\subsubsection{Defuzzification}

In defuzzification step, a crisp value is extracted from fuzzy set. For this purpose, the centroid of area strategy is taken for defuzzification in our fuzzy inference decision-making system. The defuzzifier process is based on the following equation 1 :

$$
R=\frac{\sum_{\text {All Rules }} x_{i} \times \beta\left(x_{i}\right)}{\sum_{\text {All Rules }} \beta\left(x_{i}\right)}
$$

where $R$ shows the degree of decision making, $x_{i}$ is the fuzzy variable and $\beta\left(x_{i}\right)$ is its membership function.

\subsection{Route discovery process}

In FAST, a route discovery (RD) process is initiated when a source node needs to determine a route for destination node, control algorithm diagram of FAST

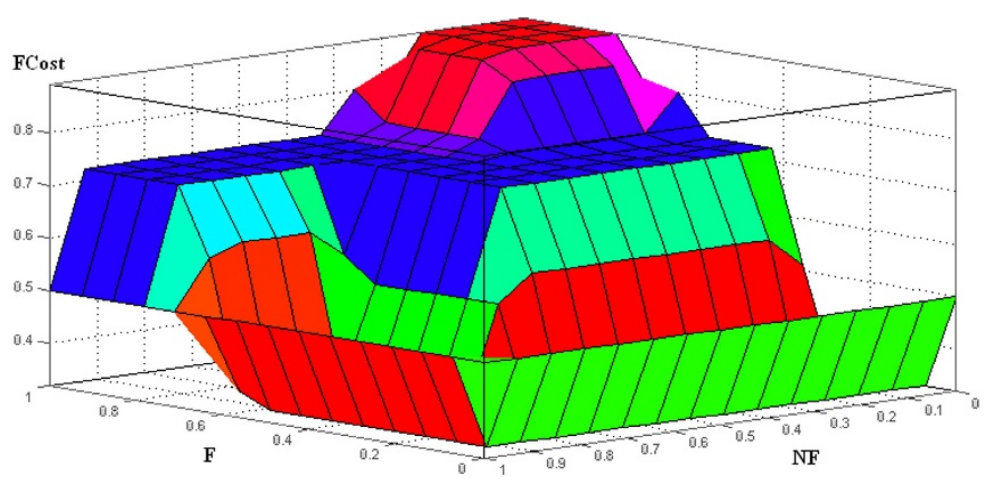

Figure 6 Correlation between input variables (friends and non-friends) and output (fuzzy-cost). 


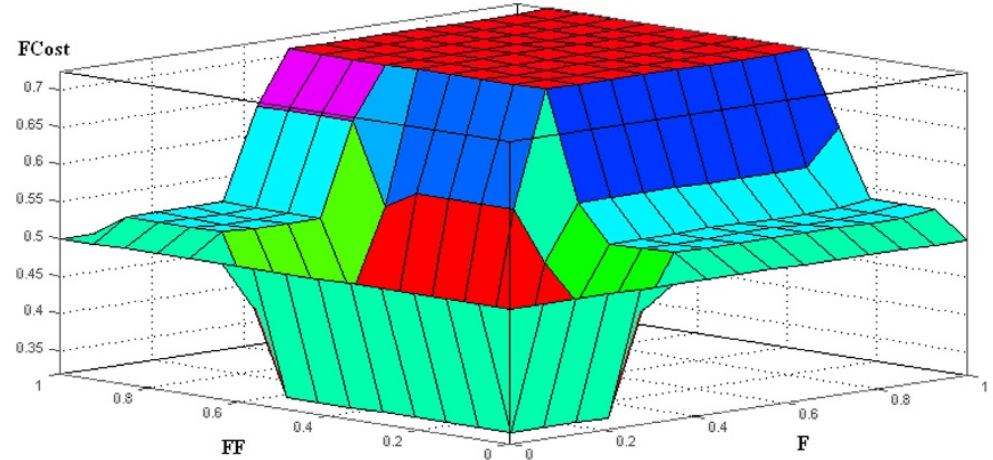

Figure 7 Correlation between input variables (friends and friends-of-friends) and output (fuzzy-cost).

protocol is illustrated in Figure 8. The source node creates a RD packet and the header of RD packet includes the address of source node, address and location of destination node, intersection ID, road segment ID, neighbor's ID, TTL and a sequence number. The source node starts flooding a RD packet until TTL value expired to discover a best route toward the destination. Lee et al. [3] suggested two ways to determine the road-density information of the network including road-side wireless sensors and each node broadcasts traffic information of

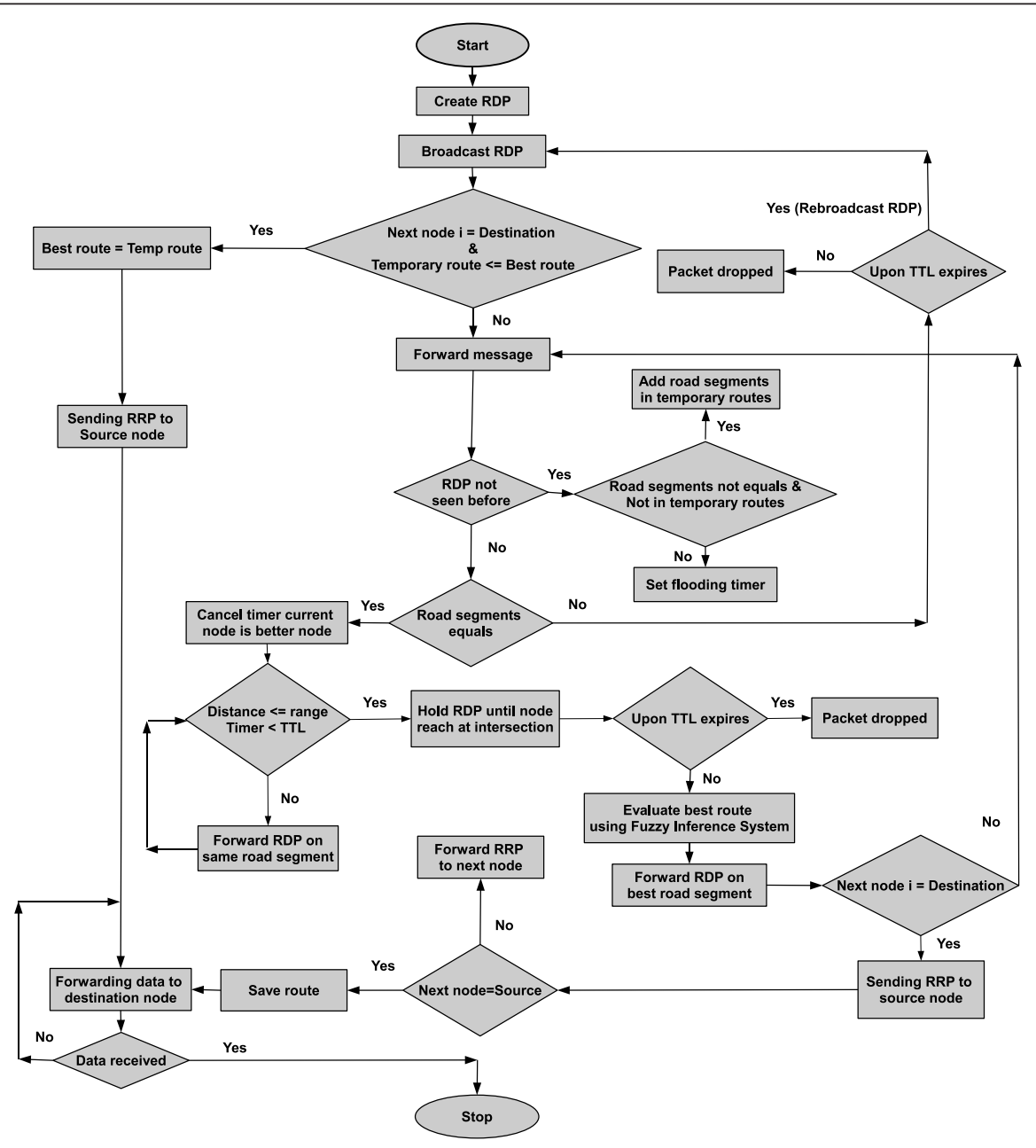

Figure 8 Control algorithm diagram of FAST protocol. 
itself and neighboring nodes. Although, the deployment of road-side wireless sensors needs major changes in the current city structure. We adopt the second method that was initially proposed to develop LOUVRE in [3]. This method is further described with the help of city scenario in the following paragraph. The flooding method is a useful method to compute the road-density information of current and next road segments. The flooding in this way may have a scalability problem and congested the sensitive VANET. Because whenever a node requests a RD packet, it sends a message that passes through potentially every node in the network. It is not a big problem, if the network is small. However, in case of large networks, like VANET, the designed protocol cannot scale with the size of the network and it can be extremely wasteful, especially if the destination node is relatively close to the source node.

To solve this broadcasting storm problem, we have used an improved flooding method that initially proposed in [19] and later improved in [5]. When any node receives a RD packet from neighbor node, it first checks the source address and sequence number from routing table, if this node already exists in routing table, it simply discarded. Upon receiving a new RD packet, instead of directly rebroadcasting this packet the node holds the packet for particular period of time inversely proportional to the distance between itself and the sending node. When this time expires, the node only re-broadcasted a RD packet, if it did not observe that this packet was already re-broadcasted by farther-away node located on the same street. Using this approach, the fartheraway nodes can rebroadcast the RD message first, thus we get the faster progress and less traffic overhead in the networks.

Figure 9 illustrates the RD process in urban scenario. A source node $S$ creates and broadcasts a RD message to neighbor nodes $N_{1}$ and $N_{2}$, and these nodes forward message to their neighbor nodes and so on until RD packet reach at destination node $D$. Each node maintains a routing table which includes, source and destination IP addresses and locations, road segments ID, intersection ID, neighbor's ID, sequence number, and hope count. A GPS is also used to get updated mobility information on each road segments and intersections. The road-density information is accordingly updated when any node leaves road segment and enters in other road segment. As shown in Figure 9, there are five nodes including one friend, three friends-of-friends, and one non-friend, on the road segment between and at intersections $I_{1}$ and $I_{5}$. The neighbors nodes $N_{1}$ and $N_{2}$ receive the packet at intersections $I_{1}$, but only $N_{1}$ will rebroadcast it in the improved flooding mechanism. Before this re-broadcast, $N_{1}$ appends intersection $I_{1}$ to the route in header of the packet.

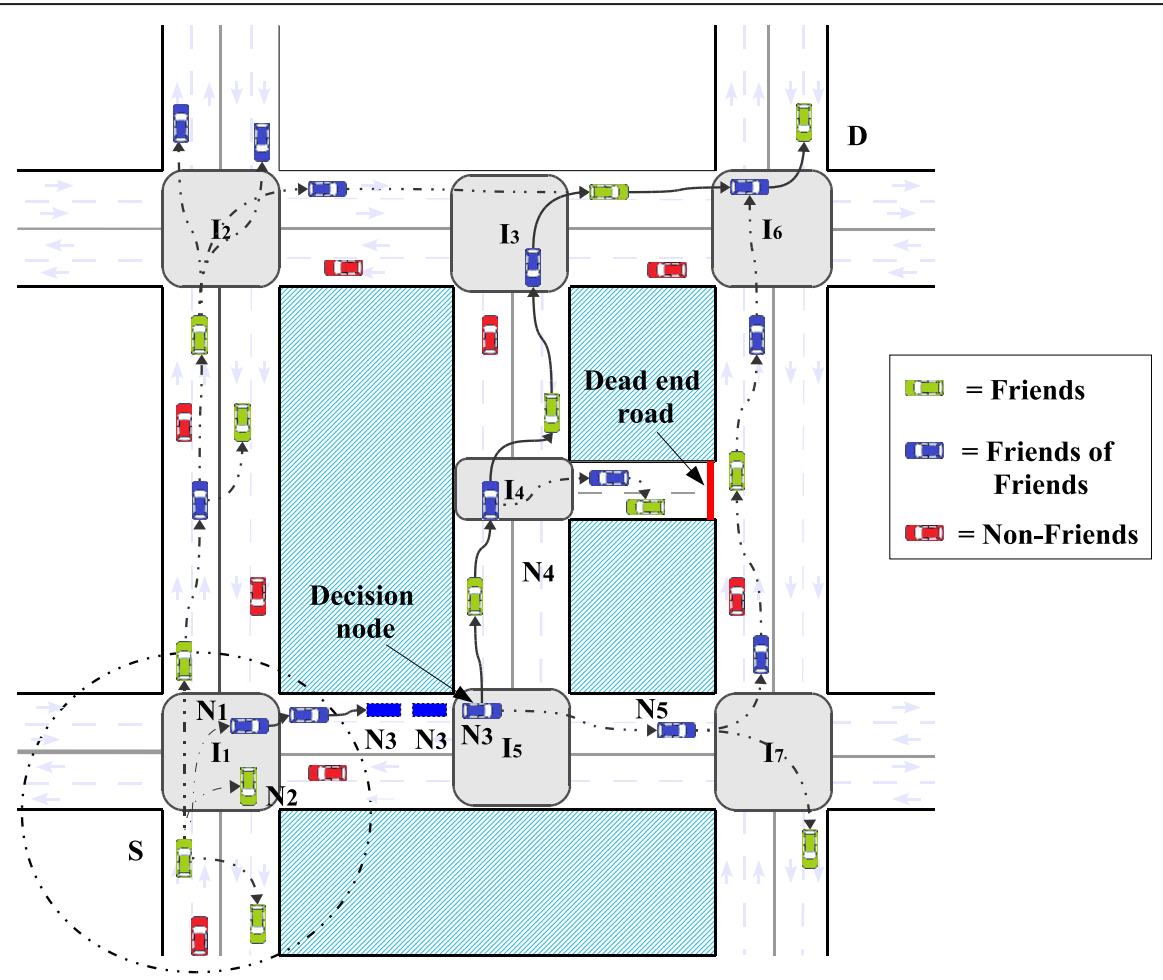

Figure 9 FAST RD process in urban scenario. 
However, when $N_{3}$ receives the RD packet, it will not update the route because $N_{3}$ is located on the same road segment with $N_{1}$. Node $N_{3}$ is close to the intersection $I_{5}$ and it will not forward RD packet across intersection $I_{5}$ to node $N_{5}$. Node $N_{3}$ holds a packet until it reaches at intersection $I_{5}$ and now $N_{3}$ become a decision-making node. At this point, $N_{3}$ get the global knowledge of real-time vehicular traffic using friendship mechanism by determining the number of nodes on next road segments. The node $N_{3}$ selects $I_{5} I_{4}, I_{4} I_{3}$ and $I_{3} I_{6}$ routes (solid arrows in Figure 9) because of the high density node and traffic flow rates. Each decision-making node at intersection calls prior global knowledge until reach the destination node $D$. The node-density information on each road segments is shown in Table 2. Also note that dead-end roads at intersection $I_{4}-D E$ will be discarded. Finally, the RD packet reaches at destination node $D$ through $I_{1}, I_{5}, I_{4}, I_{3}$ and $I_{6}$. The destination node $D$ may also receive RD packet from other nodes, the destination node $D$ always selects better quality route. If the TTL values in the RD message do not receive any reply within a certain threshold, then the destination node is considered as unreachable node, and all messages queued are removed for this destination.

\subsection{Route reply}

When the destination node receives a RD packet, it creates a route reply (RR) packet to send for the source node. As the RR packet passes through intermediate nodes, the routing tables of these nodes are updated accordingly, so that in the future, the messages can be routed through these nodes to the destination. The RR packet header includes the address and location of source node, address of destination node and shortest path length. The RR packet is forwarded based on best possible route and according to Table 2 the best possible route is $I_{6} \Rightarrow I_{3} \Rightarrow I_{4} \Rightarrow I_{5} \Rightarrow I_{1}$, as depicted in Figure 9. Also, it is possible for the RD originator to receive a RR packet from more than one node. In such cases, the $\mathrm{RD}$ originator will update its routing table with the

Table 2 Scenario of vehicular density information at and between intersections

\begin{tabular}{llll}
\hline Number & RS ID & Road segments & Node density \\
\hline 1 & id12 & 11,12 & 8 \\
2 & id23 & 12,13 & 3 \\
3 & id15 & 11,15 & 5 \\
4 & id57 & 15,17 & 2 \\
5 & id54 & 15,14 & 3 \\
6 & id43 & 14,13 & 4 \\
7 & id4D & 14, DE & 3 \\
8 & id36 & 13,16 & 4 \\
9 & id76 & 17,16 & 5 \\
\hline
\end{tabular}

most recent routing information, it uses the route with the greatest destination sequence number. We have used the node density on the road segments to measure the quality of routes. The source node starts sending data packets, when it receives RR packet.

\subsection{Route maintenance}

It has already been discussed in literatures $[13,16,20-22]$, due to high speed of vehicles the topology of VANETs has changed in few seconds and network is frequently disconnected. Route maintenance is one of the most important phases in VANET routing. FAST updates the existing routes dynamically according to the source and destination movements. The routes are updated when nodes move out of the range or move to other intersections. The dynamic global knowledge of real-time vehicular traffic is used to update routes. This process helps us to get the realtime vehicular traffic information. For example, as depicted in Figure 9 if node $S$ moves to next road segments through intersection $I_{1}$ and node $N_{2}$ moves out of the range of node $S$, then list of global knowledge parameters are accordingly updated. When node cannot find any forwarding node the route error is occurred. This route error packet is sent to source node $S$ and new RD packet is generated with certain TTL.

\section{Performance evaluation}

The performance of FAST is compared with the most related and widely used geographical and topologybased VANETs routing protocols such as GPSR [1], GPCR [2], RBVT-R [5] and GyTAR [4]. A brief review of how each of these protocols operate is given as follows. GPSR is a geographical routing protocol which forwards data packets using greedy forwarding from the source node to the destination node. When a node cannot find a neighbor node closer to the destination position than itself, a recovery strategy based on planar graph traversal is applied. Similarly, GPCR [2] is an enhancement of GPSR routing protocol that utilizes the fact that the urban street map naturally forms a planar graph. If the nodes are in the street a restricted greedy routing is used and if the nodes are at intersection the repair strategy decides which street the data packet should follow next (by right-hand rule). RBVT-R is a topology-based reactive routing protocol which creates paths containing the successions of road intersections with high probability and network connectivity using real-time vehicular traffic information. GyTAR used traffic-information before establishing routes to handle intersection and dead-end roads, same as FAST has also addressed these problems. GyTAR is an intersectionbased geographical greedy traffic-aware routing protocol 
which finds best routes in urban environments. It creates routes from source to destination based on sequence of connected intersections.

\subsection{Simulation setup}

This Section presents the simulation setup used to evaluate the performance of FAST. The area of Suffolk city map $(940 \mathrm{~m} \times 750 \mathrm{~m})$ used in with and without obstacles scenarios extracted from the TIGER Line database of the US Census Bureau [23], as shown in Figure 10. This map has many intersections and deadend roads which is most appropriate to test the performance of proposed FAST. The parameters used in simulation are defined in Table 3 . The SWANS++ simulator [24] is used which is the most scalable and efficient in memory usage network simulator. During simulation, each node equipped with a GPS receiver, a navigation system that maps GPS positions on roads to locate nodes positions and digital maps extracted from Tiger Line Database. The RAndom Waypoint mobility model with origin-destination (OD) pairs (STRAWOD) by Choffines and Bustamante [25] is used for node mobility. The STRAW have realistic vehicular mobility, contains efficient car following and lane changing model, and real-time traffic controller. The total simulation time for single flow was 300 s which is reasonable with the used area of map and number of nodes. However, the first 60 s of simulation are discarded to get more accurate node movements. During this warm-up period each mobile node will start moving properly. The IEEE $802.11 \mathrm{~b}$ with DCF standard at MAC layer was used for the wireless configuration. The radio range was set to $250 \mathrm{~m}$ for 100,150 and 200 nodes. The nodes were placed on the map using the random placement model and experiment was repeated

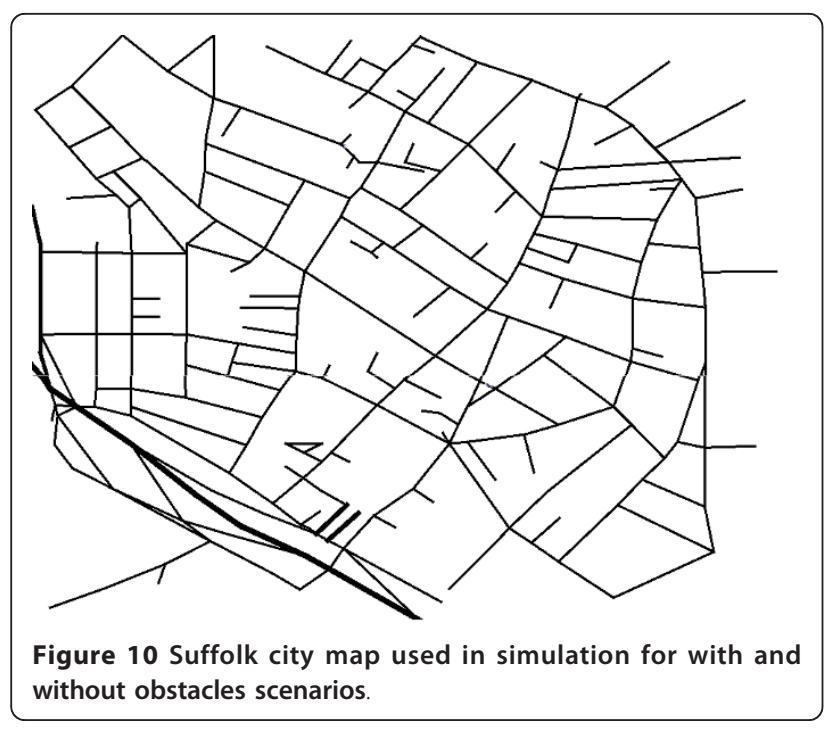

Table 3 Parameter values used in simulation for proposed FAST

\begin{tabular}{cl}
\hline Parameter & Value \\
\hline Simulation dimension & $940 \mathrm{~m} \times 750 \mathrm{~m}$ \\
Simulation area & $701528.75 \mathrm{~m}$ \\
Number of vehicles & $100-150-200$ \\
Number of CBR sources & $1-20$ \\
CBR rate & $0.5-5 \mathrm{Pkt} / \mathrm{s}$ \\
CBR packet size & 1024 \\
Transmission range & $250 \mathrm{~m}$ \\
Simulation time & $300 \mathrm{~s}$ \\
Vehicle velocity & $20-60 \mathrm{~m} / \mathrm{h}$ \\
MAC protocol & IEEE 802.11b DCF \\
Data packet size & $1052 \mathrm{bytes}$ \\
Obstacles & With and without
\end{tabular}

for 15 flows. In addition, the values of exponent for path loss formula and standard deviation for log-normal shadow fading set to 2.8 and 6.0 , respectively. In each experiment ten source and destination nodes pairs with different CBR and UDP packets are selected randomly. With the above-mentioned simulation setup, the three experiments run using the evaluation parameters PDR, average delay and average path length.

\subsection{Metrics}

The performance of the routing protocols was evaluated by varying numbers of concurrent flows, node densities and CBR data rates. PDR, average delay and average path length are the most straightforward methods of evaluating the application's performance. The metrics used to assess the performance are as follows:

- Packet delivery ratio: PDR calculates the number of data packets sent by the source node and how much data packets (in \%) the destination node successfully received. The duplicated data packets are not included that were generated by loss of acknowledgments at the MAC layer. The PDR shows the ability of the routing protocols to transfer vehicle-to$\mathrm{X}$ data packets successfully.

- Average delay: The average delay calculates the total time a message was posted by the source to destination node. The average delay characterizes the latency generated by the routing protocols.

- Average path length: This evaluation metric calculates the number of hops which take part in the data packet forwarding from source to destination nodes. The hop count is used to determine the quality of path. This metric is used to verify if there is a correlation between the path length, average delivery ratio and average delay, respectively. 


\subsection{Simulation results in urban environment (with obstacles scenario)}

Figure 11a-c shows the PDRs of FAST, RBVT-R, GyTAR, GPCR and GPSR VANET routing protocols. The PDR was calculated with 15 flows by varying three parameters such as the Constant Bit Rate (CBR) data range from 0.5 to 5.0 packets per second, parallel UDP flows, and the network densities were 100, 150 and 200 nodes. Figure $11 \mathrm{a}-\mathrm{c}$ shows the PDR of proposed FAST is about 12, 16, 28 and 32\% higher than RBVT-R, GyTAR, GPCR and GPSR respectively. It can also be observed from all cases that PDR increases when packet rate increases, which shows the protocols can transfer more data packets in the network. As depicted in Figure $11 \mathrm{~b}, \mathrm{c}$ when node density is higher (i.e., 150 and 200 nodes), RBVT-R has better performance as compared to GyTAR in some cases. This is partially due to geographical forwarding method that forwards data packets more quickly in dense networks. The PDR of GyTAR is about $16 \%$ less than FAST protocol. GyTAR divided the area between intersections into small group (called cells). GyTAR used greedy forwarding scheme to select next node in the streets. The selection procedure to find the best candidate node is probably the reason for the low accuracy in GyTAR.

The GPSR and GPCR are more affected in the presence of obstacles and PDR are consistently less than other protocols. The performance of FAST protocol is $16-28 \%$ higher than GPCR and GPSR. This is because of the local maxima frequently encountered and the data packets rerouted that may cause more packets dropped. Although, the PDR of GPCR are slightly higher in some cases (as depicted in Figure 11a-c. There are two reasons for these results. First, it is likely that there are non-empty intersections with enough vehicles to forward messages. Second, the packet did not stuck in local maxima. Figure 12a-c shows that the FAST has about 20, 30, 65 and 80\% lower average delay than GyTAR, RBVT-R, GPCR and GPSR routing protocols. The average delays of FAST are about 2.5 and $2.3 \mathrm{~s}$ when nodes densities are 100 and 150. The delay further decreased to less than $2.0 \mathrm{~s}$ when node density increases to 200 nodes. This is because in the proposed FAST the nodes do not forward the message across the

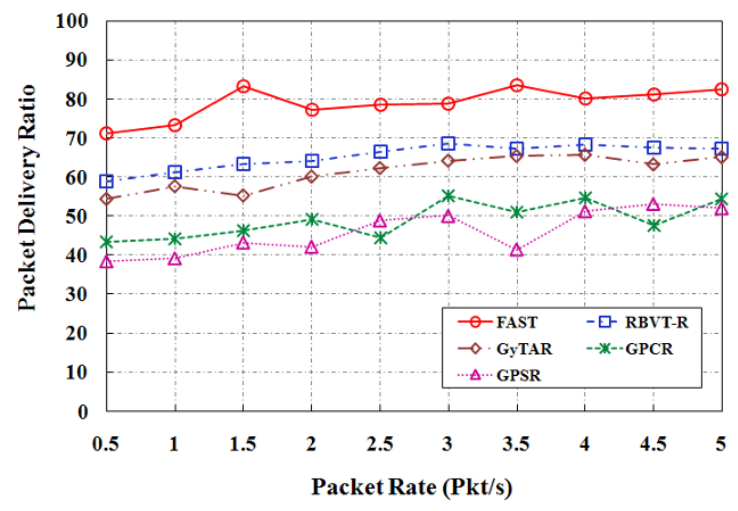

(a) PDR using 100 nodes

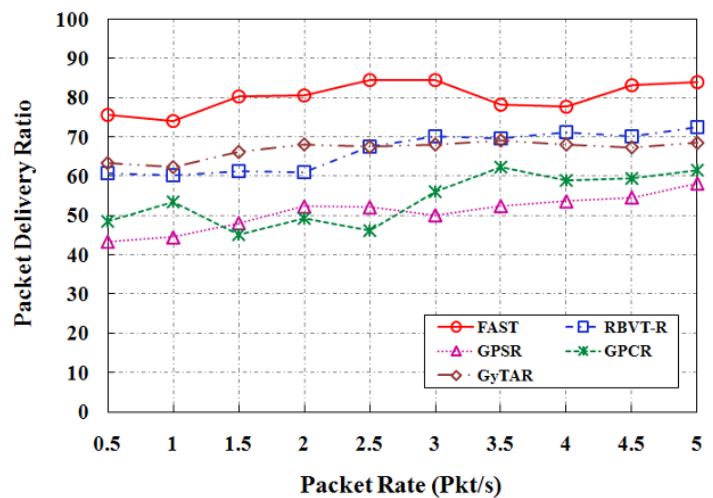

(b) PDR using 150 nodes

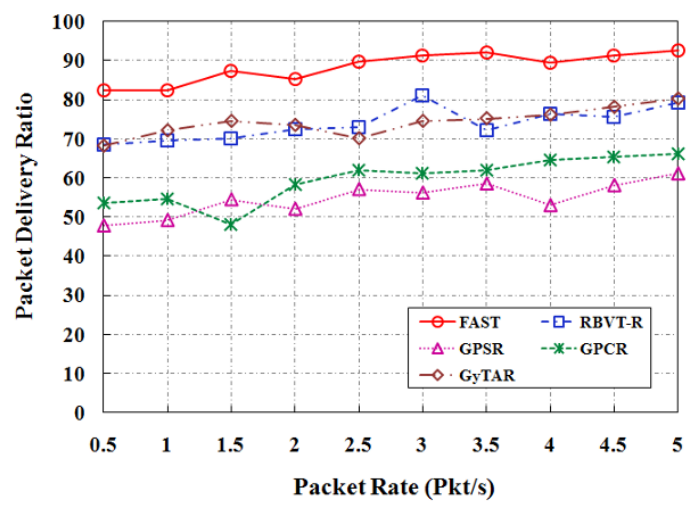

(c) PDR using 200 nodes

Figure 11 Simulation results of PDR for FAST, RBVT-R, GyTAR, GPCR, and GPSR with obstacles using different CBR and node densities. 


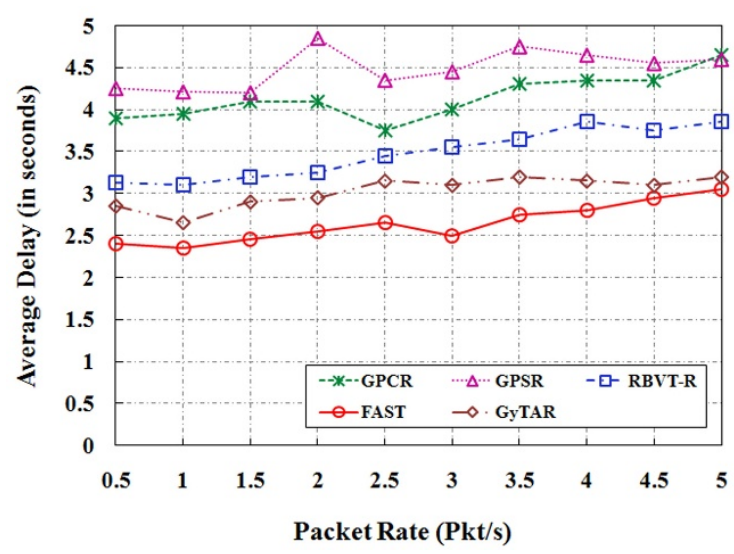

(a) Average delay using 100 nodes

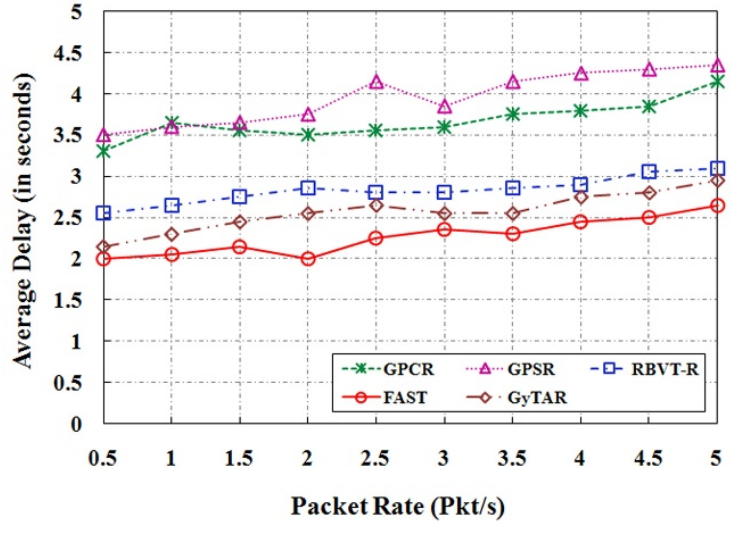

(b) Average delay using 150 nodes

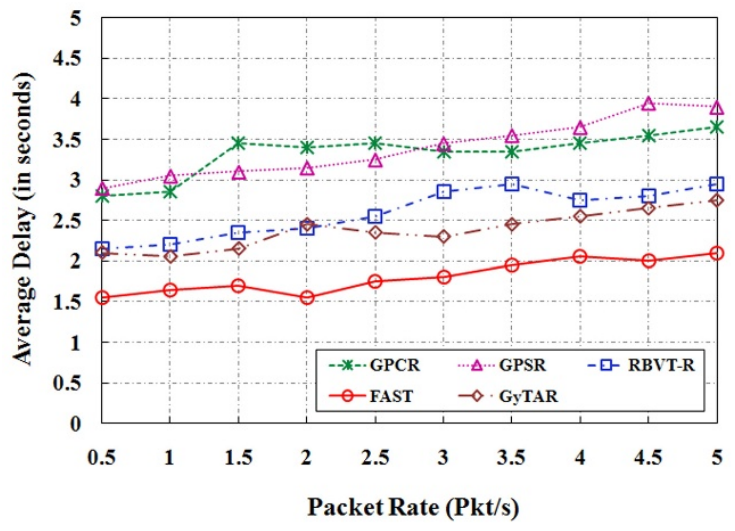

(c) Average delay using 200 nodes

Figure 12 Simulation results of average delay for FAST, RBVT-R, GyTAR, GPCR, and GPSR with obstacles using different CBR and node densities.

intersection that may cause long time to reach at destination node. The average delay of FAST decreases when node densities are increased in all cases. The main reason is that the routes remain active for longer periods of time as number of nodes increases. The source node repairs the route and fewer packets need to be buffered.

GyTAR has 20\% higher end-to-end delay than FAST in case of lower node density, 100 nodes, as shown in Figure 12a. GyTAR faces the problem of local optimum in sparse network as the next forwarding node might not be close to the next anchor. The average delays of RBVT-R are about $0.75 \mathrm{~s}$ higher than FAST protocol in all cases, as depicted in Figure 12a-c. Similarly, the average delays of GPCR and GPSR are apparently higher than other protocols in first two cases, in Figure 12a,b. However, in case of dense network the difference is reduced about 1s, as shown in Figure 12c. This is because of GPCR and GPSR forward data packets between intersections based on the location of destination node. There are two side effects of this approach such as (1) it might be possible that the road segments are congested and overall quality of communication suffers significantly, and (2) the data packets forward across the intersection that may take high delay.

The number of hops received at destination for all protocols are illustrated in Figure 13a-c. FAST received about 20,35, 40 and $50 \%$ less number of hops as compared to GyTAR, GPCR, GPSR and RBVT-R, respectively, in all cases. We can observe in all cases that the GyTAR have slightly high number of hops count than FAST. The greedy forwarding methods used in this protocol that forward data packets on road segments need some improvement for more accurate results. FAST has significance difference of hops count than GPCR in all cases, as depicted in Figure 13a-c because the presence of cross-links between source to destination cause zero hop count contribution. When number of nodes increases, the hop count increases consistently for GPSR. In GPSR, the planarization prevents packets from making large steps to the destinations. The number of 


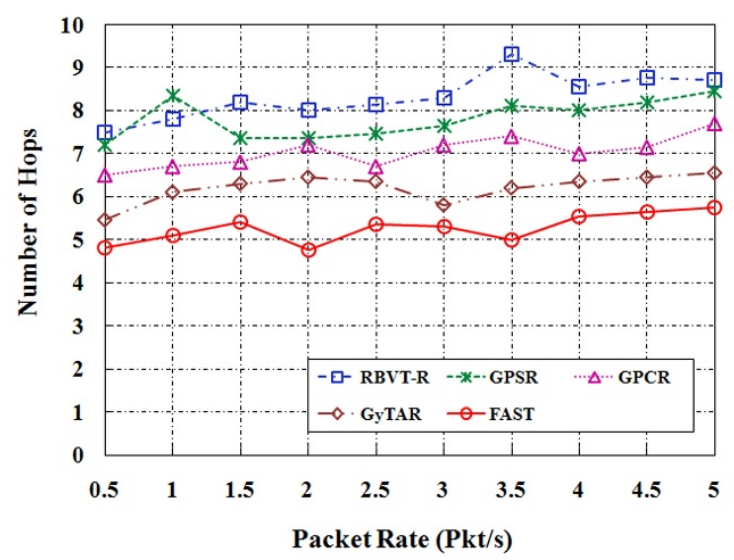

(a) Number of hops using 100 nodes

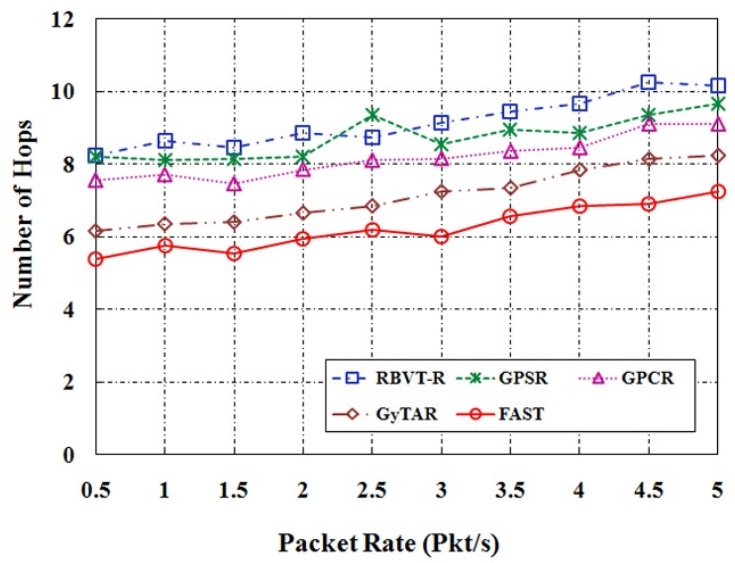

(b) Number of hops using 150 nodes

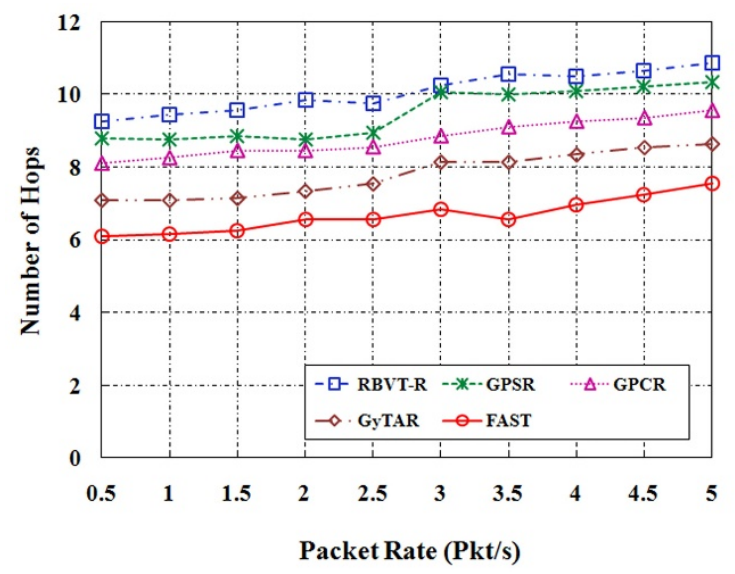

(c) Number of hops using 200 nodes

Figure 13 Simulation results of number of hops for FAST, RBVT-R, GyTAR, GPCR, and GPSR with obstacles using different CBR and node densities.

hops in all cases is higher for RBVT-R. The reason for this low result is that RBVT-R gives preference to link quality over forward progress when selecting the next neighbor node.

\subsection{Simulation results in urban environment (without obstacles scenario)}

In this scenario, the same Suffolk city map is used in with obstacle scenario. The obstacles are removed from the map in order to evaluate the performance of protocols under increased network congestion. The increase in the level of data sending rate will give us the noticeable increase in the level of contention in the network. The transmission range is set to $250 \mathrm{~m}$ for 150 nodes. With this range, it might be possible that the nodes can communicate with other nodes on the parallel streets. Figure 10 shows minimum distance between few streets less than $250 \mathrm{~m}$. The 150 nodes are placed on the map using the random placement model and repeat the experiment for 15 flows. In each experiment, ten source and destination nodes pairs with different CBR and UDP packets are selected randomly. The other simulation parameters are almost the same as described in Table 3.

To evaluate the performance of protocols, the PDR and average delay are used by increasing packet/second from 0.5 to 5 . The results for other node densities that were used in with obstacle scenario are same as for 150 nodes density. Therefore, only this scenario is used to describe the results for without obstacle.

Figure 14a shows the experimental results of FAST, RBVT-R, GyTAR, GPCR and GPSR using PDR. FAST has $5,15,17,32$ and $35 \%$ better performance of PDR than RBVT-R, GyTAR, GPCR and GPSR, respectively. The main reason for better performance of FAST is due to fuzzy-assisted friendship mechanism under dense network. As the RBVT-R use geographical forwarding method, the PDR is slightly lower than FAST. The 


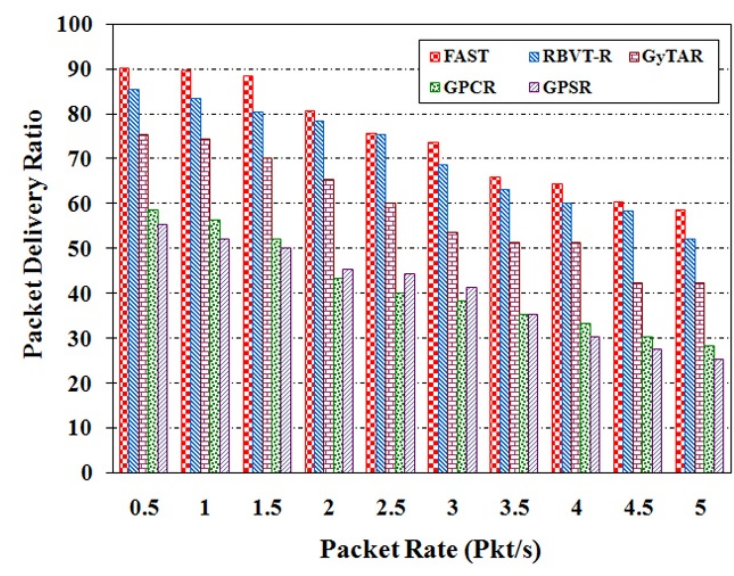

(a) Packet delivery ratio using 150 nodes without obstacles

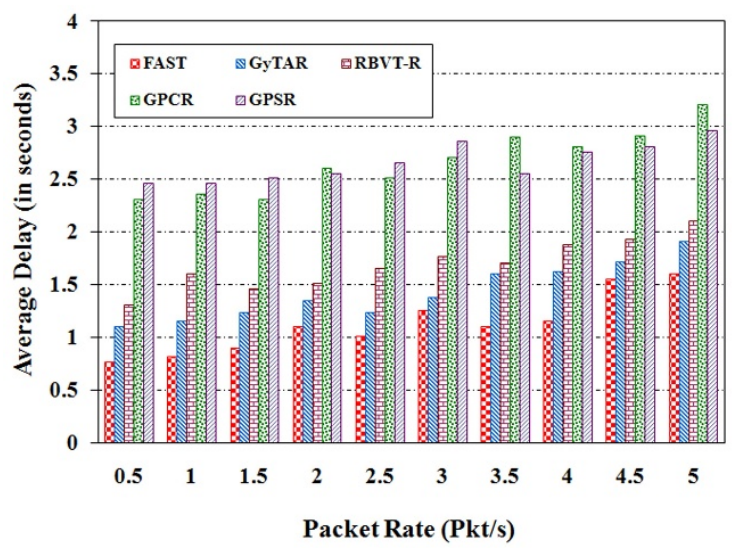

(b) Average delay using 150 nodes without obstacles

Figure 14 Simulation results of PDR and average delay for FAST, RBVT-R, GyTAR, GPCR, and GPSR without obstacles and 150 nodes density.

PDRs of GyTAR protocol is less than 15 to $20 \%$ from FAST. Similarly, the PDR's of GPCR and GPSR are lesser than other protocols under added congestion. There are two reasons for less accuracy of these protocols. First, greedy forwarding fails used in these protocols due to many dead-end roads in Suffolk city map. Second, there are some cases where the data packets reach a local maxima and forwarding mode of each packet set to perimeter forwarding that causes the packet get trapped into routing loops. Figure 14b shows the average delay of all routing protocols. As the packet rate/second increases in the networks, the average delay of all protocols increase. FAST shows the better performance of average delay than other protocols with maximum 1.5s. Average delay of other protocols increase that clearly shows high contention in the networks.

\section{Conclusion}

In this article, we proposed a FAST protocol called FAST to make better routing decisions in urban vehicular environments. Instead of simply forwarding the messages to the next available node towards destination, FAST makes dynamic routes based on friendship mechanism and fuzzy inference system for significance performance of VANET routing protocol. The simulation results in urban environment for with and without obstacles scenario show that the FAST has high PDR, low average delay, fewer hops counts as compared to some existing VANET routing protocols.

Our future study includes designing a comprehensive and fully operational misuse and anomaly intrusion detection system for FAST protocol. Also, we are currently working on the design of a mechanism to tune fuzzy membership function universes with the volatile characteristics of VANET. Our intended optimization algorithms are Artificial Bees Algorithm, Genetic Algorithm or Particle Swarm Optimization. Then, the one offer less computation overhead will be the choice in vehicular environment.

\section{Acknowledgements}

We would like to thank University of Malaya to provide fund for this research and many thanks to anonymous reviewers for their useful criticism and suggestions to improve the quality of this article.

\section{Author details}

${ }^{1}$ Faculty of Computer Science and Information Technology, University of Malaya, 50603 Lembah Pantai, Kuala Lumpur, Malaysia ${ }^{2}$ Faculty of Computer Science and Information Systems, Universiti Teknologi Malaysia, 81310 Skudai, Johor, Malaysia ${ }^{3}$ Department of Computer Science and Information Engineering, National Quemoy University, Jinning, Kinmen 892, Taiwan, ROC

\section{Competing interests}

The authors declare that they have no competing interests.

Received: 20 July 2011 Accepted: 23 November 2011 Published: 23 November 2011

\section{References}

1. B Karp, HT Kung, GPSR: greedy perimeter stateless routing for wireless networks, in MobiCom '00: Proceedings of the 6th annual international conference on Mobile computing and networking, New York, NY, USA (ACM), pp. 243-254 (2000)

2. C Lochert, M Mauve, H Fubler, $\mathrm{H}$ Hartenstein, Geographic routing in city scenarios. SIGMOBILE Mob Comput Commun Rev. 9, 69-72 (2005)

3. K Lee, M Le, J Harri, M Gerla, LOUVRE: landmark overlays for urban vehicular routing environments. in IEEE 68th Vehicular Technology Conference, 2008. VTC 2008-Fall 1-5 (2008)

4. M Jerbi, SM Senouci, T Rasheed, Y Ghamri-Doudane, Towards efficient geographic routing in urban vehicular networks. IEEE Trans Veh Technol. 58(9), 5048-5059 (2009)

5. J Nzouonta, N Rajgure, G Wang, C Borcea, VANET routing on city roads using real-time vehicular traffic information. IEEE Trans Veh Technol. 58(7), 3609-3626 (2009)

6. KC Lee, PC Cheng, M Gerla, GeoCross: a geographic routing protocol in the presence of loops in urban scenarios. Ad Hoc Netw. 8(5), 474-488 (2010). doi:10.1016/j.adhoc.2009.12.005 
7. RH Khokhar, MN Asri, MS Latiff, MA Amin, Reactive traffic-aware routing strategy for urban vehicular environments. Int J Ad Hoc Ubiq Comput (2011, in press)

8. P Bose, P Morin, I Stojmenovic, J Urrutia, Routing with guaranteed delivery in ad hoc wireless networks. Wirel Netw. 7(6), 609-616 (2001). doi:10.1023/ A:1012319418150

9. F Kuhn, R Wattenhofer, Y Zhang, A Zollinger, Geometric ad-hoc routing: of theory and practice, in PODC '03: Proceedings of the twenty-second annual symposium on Principles of distributed computing, New York, NY, USA (ACM), pp. 63-72 (2003)

10. K Lee, J Haerri, U Lee, M Gerla, Enhanced perimeter routing for geographic forwarding protocols in urban vehicular scenarios, in Globecom Workshops, 2007, pp. 1-10 (IEEE, 2007)

11. KH Chen, CR Dow, SC Chen, YS Lee, SF Hwang, HarpiaGrid: a geographyaware grid-based routing protocol for vehicular ad hoc networks. J Inf Sci Eng. 26, 817-832 (2010)

12. B Leong, B Liskov, R Morris, Geographic routing without planarization, in NSDl'06: Proceedings of the 3rd conference on Networked Systems Design \& Implementation, Berkeley, CA, USA (USENIX Association), p. 25 (2006)

13. YJ Kim, R Govindan, B Karp, S Shenker, Geographic routing made practical, in Proceedings of the 2nd conference on Symposium on Networked Systems Design \& Implementation, vol. 2. NSDI'05, Berkeley, CA, USA (USENIX Association), pp. 217-230 (2005)

14. SA Razak, SM Furnell, NL Clarke, PJ Brooke, Friend-assisted intrusion detection and response mechanisms for mobile ad hoc networks. Ad Hoc Netw. 6, 1151-1167 http://portal.acm.org/citation.cfm?id=1389584.1389880 (2008). doi:10.1016/j.adhoc.2007.11.004

15. SA Razak, N Samian, MA Maarof, SM Furnell, NL Clarke, PJ Brooke, A friend mechanism for mobile ad hoc networks. J Inf Assur Secur. 4, 440-448 (2009)

16. C Huang, I Chen, K Hu, H Shen, Y Chen, D Yang, A load balancing and congestion-avoidance routing mechanism for teal-time traffic over vehicular networks. Univer Comput Sci. 15(13), 2506-2527 (2009)

17. K Zrar Ghafoor, K Abu Bakar, M van Eenennaam, R Khokhar, A Gonzalez, A fuzzy logic approach to beaconing for vehicular ad hoc networks. Telecommun Syst 1-11 (2011)

18. E Mamdani, Application of fuzzy logic to approximate reasoning using linguistic synthesis. IEEE Trans Comput. C-26(12), 1182-1191 (1977)

19. L Briesemeister, G Hommel, Role-based multicast in highly mobile but sparsely connected ad hoc networks, in MobiHoc '00: Proceedings of the 1st ACM international symposium on Mobile ad hoc networking \& computing, Piscataway, NJ, USA (IEEE Press), pp. 45-50 (2000)

20. F Li, Y Wang, Routing in vehicular ad hoc networks: a survey. IEEE Veh. Technol Mag. 2(2), 12-22 (2007)

21. J Bernsen, D Manivannan, Unicast routing protocols for vehicular ad hoc networks: a critical comparison and classification. Pervas Mob Comput. 5, 1-18 (2009). doi:10.1016/j.pmcj.2008.09.001

22. KC Lee, $U$ Lee, M Gerla, Survey of routing protocols in vehicular ad hoc networks. in /GI Global 2010

23. Tiger: tiger/line and tiger-related products. U.S. Census Bureau, http://www. census.gov/geo/www/tiger/ (2011)

24. Swans++: Swans++ Simulator, http://www.aqualab.cs.northwestern.edu/ projects/swans+t/ (2011)

25. DR Choffnes, FE Bustamante, An integrated mobility and traffic model for vehicular wireless networks, in VANET '05: Proceedings of the 2nd ACM international workshop on Vehicular ad hoc networks, New York, NY, USA (ACM), pp. 69-78 (2005)

doi:10.1186/1687-1499-2011-178

Cite this article as: Khokhar et al:: Fuzzy-assisted social-based routing for urban vehicular environments. EURASIP Journal on Wireless

Communications and Networking 2011 2011:178.

Submit your manuscript to a SpringerOpen ${ }^{\mathcal{O}}$ journal and benefit from:

- Convenient online submission

- Rigorous peer review

- Immediate publication on acceptance

- Open access: articles freely available online

- High visibility within the field

- Retaining the copyright to your article

Submit your next manuscript at $\gg$ springeropen.com 